\title{
Using zeta-potential measurements to quantify peptide partition to lipid membranes
}

\author{
João M. Freire $\cdot$ Marco M. Domingues • \\ Joana Matos $\cdot$ Manuel N. Melo $\cdot$ Ana Salomé Veiga $\cdot$ \\ Nuno C. Santos $\cdot$ Miguel A. R. B. Castanho
}

Received: 8 October 2010/Revised: 28 November 2010/Accepted: 15 December 2010/Published online: 13 January 2011

(C) The Author(s) 2011. This article is published with open access at Springerlink.com

\begin{abstract}
Many cellular phenomena occur on the biomembranes. There are plenty of molecules (natural or xenobiotics) that interact directly or partially with the cell membrane. Biomolecules, such as several peptides (e.g., antimicrobial peptides) and proteins, exert their effects at the cell membrane level. This feature makes necessary investigating their interactions with lipids to clarify their mechanisms of action and side effects necessary. The determination of molecular lipid/water partition constants $\left(K_{p}\right)$ is frequently used to quantify the extension of the interaction. The determination of this parameter has been achieved by using different methodologies, such as UV-Vis absorption spectrophotometry, fluorescence spectroscopy and $\zeta$-potential measurements. In this work, we derived and tested a mathematical model to determine the $K_{p}$ from $\zeta$-potential data. The values obtained with this method were compared with those obtained by fluorescence spectroscopy, which is a regular technique used to quantify the interaction of intrinsically fluorescent peptides with selected biomembrane model systems. Two antimicrobial peptides (BP100 and pepR) were evaluated by this new method. The results obtained by this new methodology show that $\zeta$-potential is a
\end{abstract}

Membrane-active peptides: 455th WE-Heraeus-Seminar and AMP 2010 Workshop.

J. M. Freire - M. M. Domingues - J. Matos ·

M. N. Melo · A. S. Veiga - N. C. Santos ·

M. A. R. B. Castanho $(\bowtie)$

Instituto de Medicina Molecular, Faculdade de Medicina

da Universidade de Lisboa, Av. Prof. Egas Moniz,

1649-028 Lisbon, Portugal

e-mail: macastanho@fm.ul.pt

A. S. Veiga

Chemical Biology Laboratory, National Cancer Institute,

Frederick, MD 21702, USA powerful technique to quantify peptide/lipid interactions of a wide variety of charged molecules, overcoming some of the limitations inherent to other techniques, such as the need for fluorescent labeling.

Keywords Partition constant - Zeta-potential · Fluorescence $\cdot$ Membrane $\cdot$ Antimicrobial peptides

\section{Introduction}

Many molecules, such as several peptides and proteins, exert their effects at the cell membrane level, which makes necessary investigating their interactions with lipids to study their mechanisms of action and side effects (Reddy et al. 2004; Dawson and Liu 2008; Hancock and Chapple 1999). Liposomes or lipid vesicles are useful as a membrane model system because they mimic the cell membrane properties in a wide range of interactions. The molecular in-depth location and orientation in lipid bilayers of a given molecule can be studied using liposomes and other lipid models (Lopes and Castanho 2005; Troeira Henriques et al. 2007; Castanho et al. 1996; Castanho and Fernandes 2006b). Determination of molecular lipid/water partition constants $\left(K_{p}\right)$ is frequently used to quantify the extension of the interaction (Santos et al. 2003; Ribeiro et al. 2010). The determination of this parameter has been achieved by using different methodologies, such as UV-Vis absorption spectrophotometry, fluorescence spectroscopy and $\zeta$-potential (zetapotential) measurements. These three techniques are among the most used to calculate $K_{p}$ without physical separation of the "lipid phase" from "aqueous phase", as happens in chromatographic techniques (Santos et al. 2003; Matos et al. 2010; Ribeiro et al. 2010). Another advantage of these techniques is the use of optical signals, which are 
noninvasive to the system under study. However, both UVVis spectrophotometry and fluorescence spectroscopy require molecules that have chromophores or fluorophores in their structures (Santos et al. 2003). At variance, application of $\zeta$-potential-based methodologies only require charged molecules in the system in order to measure surface charge alterations when molecules interact with liposomes (Kaszuba et al. 2010).

Polyelectrolyte particles in a suspension attract ions to their surface. These ions form a layer covering the particle surface, commonly named the Stern layer (Fig. 1). Another layer outside the Stern layer is also formed, where ions diffuse more freely. Within the diffuse layer there is a notional boundary inside which the particle forms a stable entity. When a particle moves, in the presence of an electrical field, the ions within this boundary move with it. In the diffuse boundary, the ions do not move concomitantly to the scattering particle. The electric potential that exists at this boundary is called the $\zeta$-potential (Domingues et al. 2008; Kaszuba et al. 2010). This potential determines the electrophoretic mobility of the particles in solution on the progression towards the electrode of opposite charge in the presence of an electric field. Viscous forces oppose to the movement of the particles until equilibrium, when a constant velocity is reached. The electrophoretic mobility can be calculated through the analysis of electrophoresis performed with different experimental conditions. Alternatively, it can be determined by laser Doppler velocimetry using dynamic light scattering-based $\zeta$-potential

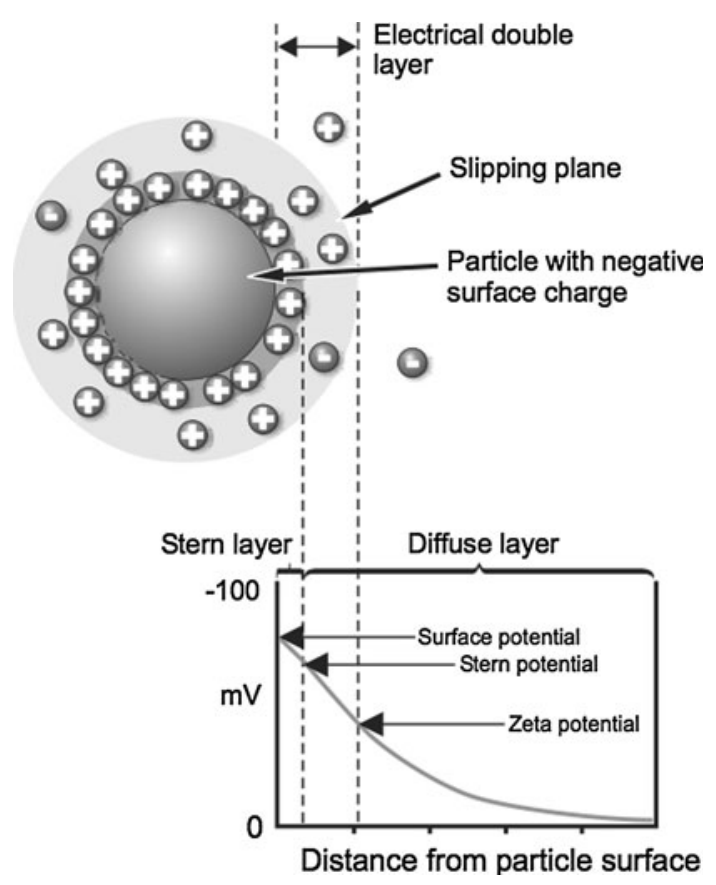

Fig. 1 Schematic representation of the double layer that surrounds a particle in aqueous medium. The potential that exists at the slipping plane is known as the $\zeta$-potential. Taken from (Malvern 2007) measurement devices, in which particle velocity is related to frequency, measured by intensity fluctuation of the scattered light. However, the sensitivity of the Doppler effect to the low mobility of larger particles is very low, making it difficult for the electrophoretic mobility to be calculated. Some newer devices can use a different method for calculating electrophoretic mobility. This method was developed in the early 1990s by Miller et al. (1991) and is based on phase shift analysis, where differences in phases between an unshifted reference beam and the samplescattered beam are analyzed with higher sensitivity. This phase shift is related to the position of the particle. Hence, the mean phase change with time yields the electrophoretic motion (Miller et al. 1991; Tscharnuter 2001). Using Henry's relation (Delgado et al. 2007), it is possible to calculate the $\zeta$-potential of the particle:

$U_{\mathrm{E}}=\frac{2 \varepsilon z f(\mathrm{ka})}{3 \eta}$

In this equation, $z$ is the $\zeta$-potential, $U_{\mathrm{E}}$ the electrophoretic mobility and $f(\mathrm{ka}$ ) the Henry's function (Delgado et al. 2007; Kirby and Hasselbrink 2004). Thus, the $\zeta$-potential enables the assessment of the membrane potential, which in turn depends on the adsorption or insertion of charged molecules in lipid bilayers. This allows quantifying the extent of the binding between charged molecules and lipid membranes, from which the $K_{p}$ can be obtained.

In this work we derived and tested a mathematical model to determine the $K_{p}$ from $\zeta$-potential data. We compared the values obtained with those obtained by fluorescence spectroscopy, which is a regular technique used to quantify peptide/lipid interactions in selected biomembrane model systems (Santos et al. 2003). Two antimicrobial peptides (AMP) were studied by this new method: BP100 (KKLFKKILKYL-NH2), a short cationic cecropin A-melittin hybrid with activity against several plant pathogenic gram-negative bacteria (Alves et al. 2010; Ferre et al. 2009), and pepR (LKRWGTIKKSKAINVLR GFRKEIGRMLNILNRRRR), a peptide derived from the putative RNA-binding domain of the dengue virus capsid protein (Alves et al. 2010). The results obtained by this new methodology show that $\zeta$-potential is a powerful technique to quantify protein/lipid interactions of a wide variety of charged molecules, overcoming some of the limitations inherent to other techniques.

\section{Materials and methods}

\section{Materials}

2-(4-(2-Hydroxyethil)-1-piperazinyl)-ethanosulfonic acid (HEPES), sodium chloride, chloroform, dimethlyl sulfoxide 
(DMSO), ethanol (Spectroscopic grade) and L-tryptophan from Merck (Darmstadt, Germany) were used. Phospholipids 1-palmitoyl-2-oleoyl-sn-glycero-3-phosphocholine (POPC), 1-palmitoyl-2-oleoyl-sn-glycero-3-phospho-L-serine (POPS) and 1-palmitoyl-2-oleoyl-sn-glycero-3-(phosphor-rac-(1-glycerol)) (POPG) were from Avanti Polar Lipids (Alabaster, AL). Unless otherwise stated, all solutions were prepared in HEPES buffer pH 7.4 (10 mM HEPES, $150 \mathrm{mM}$ of $\mathrm{NaCl}$ ).

Preparation of lipid vesicles

Large unilamellar vesicles (LUV) were prepared by extrusion of multilamellar vesicles (MLV) using a miniextruders from Avanti Polar Lipids (Alabaster, AL) and used as biological membrane models (Santos and Castanho 2002b; Mayer et al. 1986). MLVs were obtained from the hydration with $\mathrm{pH}$ 7.4 HEPES buffer of vacuum-dried phospholipid films that were previously dissolved in chloroform (Mayer et al. 1986). The extrusion protocol was carried out using 1001RN syringes (Hamilton, Reno, NV) with 13-mm-diameter membrane filters (400-nm pore) and 100-nm-pore polycarbonate 10-mm-diameter membranes from Nucleopore-Wathman (Kent, UK), leading to the production of 100-nm-diameter vesicles.

Determination of the partition constant by fluorescence spectroscopy

The formalism for the determination of the partition constant by spectroscopic methods has been described elsewhere (Santos et al. 2003; Ribeiro et al. 2010; Castanho and Fernandes 2006a). Briefly, the partition constant can be formulated by:

$K_{P}=\frac{[P]_{L}}{[P]_{W}} \Leftrightarrow K_{P}=\frac{\frac{n_{s, L}}{V_{L}}}{\frac{n_{s, W}}{V_{W}}}$

In Eq. $2, K_{P}$ represents the partition constant, $n_{s, i}$ and $[P]_{i}$ are respectively the moles of solute and the peptide concentration in each environment $i$ ( $i=W$, water; $i=L$, lipid). From Eq. 2 the fraction of membrane-bound $\left(X_{L}\right)$ solute can be related to $K_{p}$ (Santos et al. 2003):

$X_{L}=\frac{K_{P} \gamma_{L}[L]}{1+K_{P} \gamma_{L}[L]}$

$X_{L}$ represents the fraction of peptide that is bound to the lipid, $[L]$ the phospholipid concentration (considering that the volume is only given by the aqueous volume), and $\gamma_{L}$ is the molar volume of the lipid.

Fluorescence intensity $\left(I_{f}\right)$ can be used to study the interaction of fluorophore-bearing molecules, such as tryptophan-containing peptides, with lipid bilayers, as long as the fluorophore is sensitive to changes in its micro-environment, namely, changes in polarity. It is expected that, when in contact with a lipid bilayer, there will be an increase in the tryptophan fluorescence quantum yield, for instance. The variation of the fluorescence intensity with $[L]$ is a linear combination of the contribution of the molecules in the aqueous or on the lipid phase. The value of $K_{p}$ is given by (Santos et al. 2003):

$\frac{I_{f}}{I_{W}}=\frac{1+K_{P} \gamma_{L}[L] \frac{I_{L}}{I_{W}}}{1+K_{P} \gamma_{L}[L]}$

where $I_{W}$ and $I_{L}$ are the fluorescence intensities arising when all the peptide is in the aqueous and the lipid phases, respectively.

Fluorescence measurements were performed on a FS920 fluorescence spectrophotometer FL920 (Edinburgh Instruments, Livingston, UK), using 0.5-cm quartz cells (Hellma, Jena, Germany). The concentration of the fluorophore was used so that the solution had 0.1 of maximum absorbance at the excitation wavelength, optimizing the signal/noise ratio and minimizing inner filter effects. All emission spectra were obtained in the 300-450-nm range, with an excitation wavelength of $280 \mathrm{~nm}$.

The partition experiments using steady-state fluorescence spectroscopy were carried out by titrating a solution of pepR $(36 \mu \mathrm{M})$ or BP100 $(1.5$ or $10 \mu \mathrm{M})$ with a $15-\mathrm{mM}$ (pepR) or 3.5-mM (BP100) stock suspension of LUV. All the measurements were carried out at room temperature. The system was incubated after each lipid addition for 10 min before fluorescence measurement. By a non-linear fitting of the observable $I_{\mathrm{f}}$ versus $[L]$ data, one can obtain the values for the partition constant, $K_{p}$. Spectra corrections were applied as described elsewhere (Ladokhin et al. 2000) before data analysis.

Determination of the partition constant by zeta-potential

Here we present a mathematical formalism to determine partition constants of polyelectrolytes, such as charged peptides (a characteristic shared by almost all AMP), to lipid vesicles by $\zeta$-potential measurements. The basic assumption of this method is that the particle under study can be electrophoretically displaced, which is a consequence of its global net charge. This method relies on electrostatic interactions, which have an important role in partition phenomena. $\zeta$-Potential is known to be dependent on the binding of a solute to lipid vesicles (LUV) when the net charge is affected (Domingues et al. 2008). Lipid vesicles are also good scatterers; therefore, $\zeta$-potential measurements are an appropriate approach to quantify partition constants. Titrations of lipid vesicles with peptide are performed in order to determine the partition constant 
from $\zeta$-potential measurements. When a lipid vesicle with a negative $\zeta$-potential interacts with a positively charged peptide, for instance, the $\zeta$-potential value increases, progressively approaching a negative, neutral or positive threshold.

To extract the $K_{P}$ value by this methodology, it is necessary to know in advance the net charge of the peptide and the proportion and effective charge of the ionic phospholipids in the vesicles. When a positive peptide interacts with negatively charged membrane, for instance, there is a reduction in the magnitude of the surface charge of the membrane. This reduction is proportional to the fraction of charged lipids that are neutralized, given by:

$\left|\frac{\Delta \zeta}{\zeta_{0}}\right|=\frac{n_{P L_{\text {neutralized }}^{-}}}{n_{P L_{\text {total }}^{-}}}$

where $\left|\Delta \zeta / \zeta_{0}\right|$ is the relative reduction in $\zeta$-potential, which is dependent on the global peptide concentration, $[P]$; $n_{P L_{\text {neutralized }}^{-}}$is the number of charged phospholipids that were perturbed by the peptide interaction, and $n_{P L_{\text {total }}^{-}}$is the total amount of charged phospholipids. Assuming that each nominal peptide charge, when interacting with the lipid membrane, neutralizes one nominal phospholipid charge, it is possible to replace $n_{P L_{\text {neutralized }}^{-}}$by:

$n_{P L_{\text {neutralized }}^{-}}=n_{\text {Peptide }, L} \cdot z_{\text {Peptide }}$

where $n_{\text {Peptide, } L}$ moles of bound peptide and $z_{\text {Peptide }}$ is the global charge of the peptide. Equation 5 can then be rearranged to:

$\left|\frac{\Delta \zeta}{\zeta_{0}}\right|=\frac{X_{L} z_{\text {Peptide }}}{f_{P L}[L]} \cdot[P]$

$X_{L}$ represents the molar fraction of peptide that is in contact with the membrane and is described by Eq. 3. [L] and $[P]$ are respectively the lipid and peptide concentration, and $f_{\mathrm{PL}}$ is the fraction of anionic phospholipids in the lipid mixture. Considering that a phospholipid can have more than a single charge, Eq. 7 can be rearranged to:

$\frac{\zeta_{\text {final }}}{\zeta_{0}}=1+\frac{X_{L} z_{\text {Peptide }}}{f_{P L}[L] z_{\text {Lipid }}} \cdot[P]$

where $z_{\text {lipid }}$ is the absolute charge of the lipid and $\zeta_{\text {final }}$ is the $\zeta$-potential value for each titration with peptide. Plotting $\zeta_{\text {final }} / \zeta_{0}$ versus $[P]$, a linear variation is expected, and it is possible to determine the value of $K_{P}$ from the slope using Eqs. 8 and 3.

Zeta-potential measurements were performed in a Malvern Zetasizer Nano ZS apparatus (Malvern, UK) with a backscattering detection at a constant $173^{\circ}$ scattering angle, equipped with a $\mathrm{He}-\mathrm{Ne}$ laser $(\lambda=632.8 \mathrm{~nm})$, at $25^{\circ} \mathrm{C}$. Zetasizer folded capillary cells DTS 1060 (Malvern, UK) were used in the $\zeta$-potential experiments.
Aliquots of constant $200 \mu \mathrm{M}$ of LUV were prepared to a final sample volume of $1 \mathrm{ml}$ into a sterile Eppendorf tube and then filtered into the $\zeta$-potential cuvette. Filtered $\mathrm{pH}$ 7.4 HEPES buffer was used in all the samples.

For each sample the instrument performed 20 scans $(70$ runs each), with an initial equilibration time of $15 \mathrm{~min}$, at $25^{\circ} \mathrm{C}$ and a constant voltage of $40 \mathrm{mV}$. Values of the viscosity and refractive index were set at $0.8872 \mathrm{cP}$ and 1.330 , respectively. Data analysis was processed using the instrumental Malvern's DTS software to obtain the mean $\zeta$-potential value. All experiments were performed in triplicate. Equation 8 was used to determine the $K_{p}$ value.

\section{Results and discussion}

We developed a new mathematical formalism to determine $K_{p}$ from $\zeta$-potential data. To validate this method, the $K_{p}$ values obtained for two different membrane-active peptides, pepR and BP100, were compared with those calculated from fluorescence spectroscopy-based methodologies (Santos et al. 2003). Figures 2a, b and 3 show the results obtained for pepR by $\zeta$-potential and fluorescence techniques, respectively. $K_{p}$ was also obtained for BP100 in POPC:POPG 3:1 lipidic mixtures (Fig. 2c). The $K_{p}$ values are summarized in Table 1.

In the $\zeta$-potential experiments, LUV suspensions were titrated with peptide solutions in HEPES buffer. As expected, the interaction of the positively charged peptides with negatively charged LUV causes membrane surface charge to change. Increasingly, positive $\zeta$-potential values are detected and, at higher peptide concentrations, a neutralization of the charge is observed (Fig. 2a). The increase of the $\zeta$-potential reflects the interaction of the peptides with the lipid bilayers, which progressively neutralizes the net negative charge of the phospholipid polar head group. Significant $\zeta$-potential changes are not observed when using lipid vesicles with neutral global net charge. When POPC vesicles were titrated with pepR no changes in $\zeta$-potential values were observed, being indicative of a weak or even no interaction of the peptide with zwitterionic lipids. These results show that the interaction of pepR with lipid membranes is driven by electrostatic interactions. The $\zeta$-potential values obtained for each peptide were normalized to the initial $\zeta$-potential value of the LUV suspension in the absence of the peptide $\left(\zeta_{0}\right)$. We then applied Eq. 8 to the obtained results to determine the $K_{p}$ value of each interaction (see Fig. $2 \mathrm{~b}$ and Table 1). The net charges considered were $-1,+12$ and +6 for the anionic phospholipids, pepR and BP100, respectively.

When fluorescence spectroscopy was applied to calculate $K_{p}$, a peptide solution was titrated with a LUV suspension. In Fig. 3 an increase in the tryptophan fluorescence intensity 

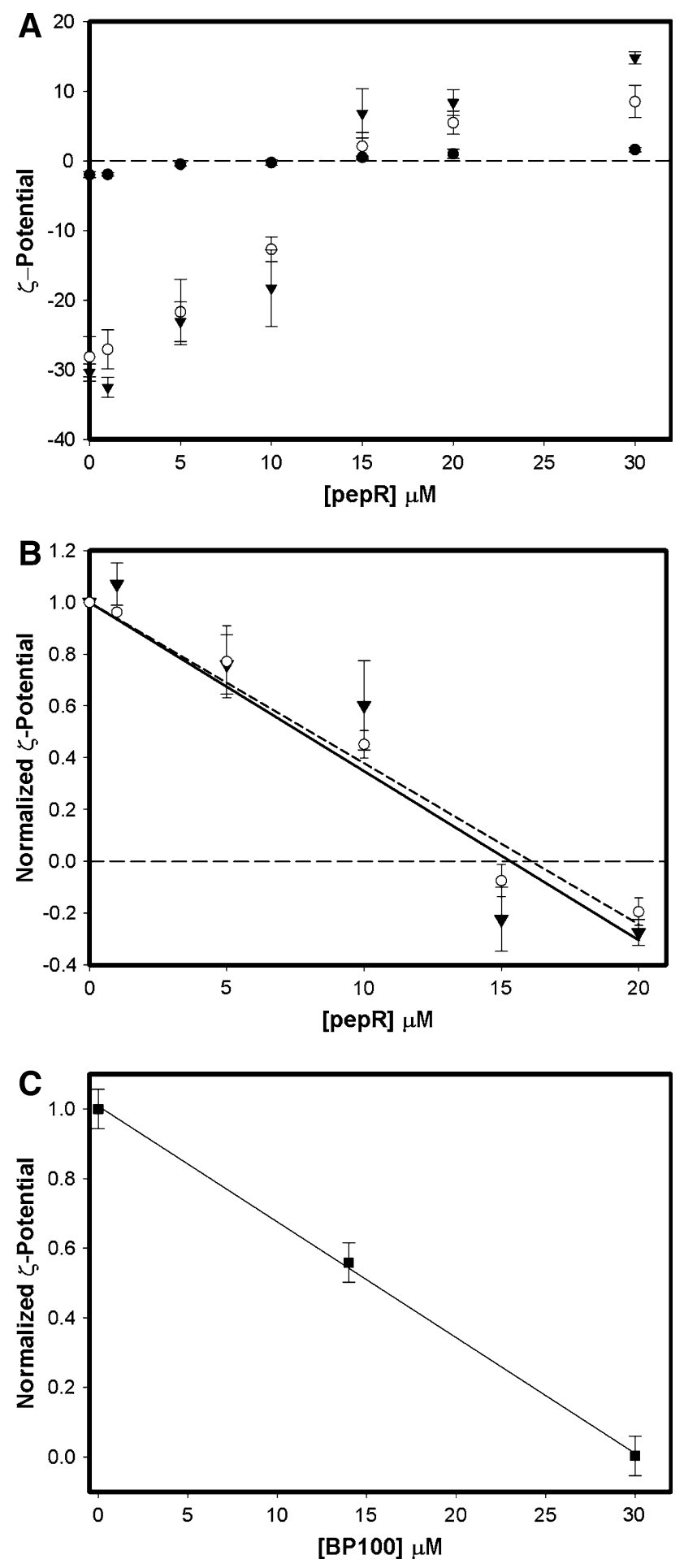

is observed due to the increasing number of molecules coming in contact with the nonpolar environment. The quantification of these interactions was done by a non-linear fitting of the obtained results with Eq. 4 (Table 1).

Both methods retrieved comparable $K_{p}$ values. $\zeta$-Potential measurements prove to be a reliable method for studying peptide/lipid interactions and for $K_{p}$ determination. Yet when
Fig. 2 Determination of the partition constant by $\zeta$-potential measurements. a $\zeta$-Potential measurements of different lipid mixtures in the presence of pepR. Filled circles POPC, empty circles POPC:POPG 4:1 and filled triangles POPC:POPG 3:2. All measurements were performed with a $200 \mu \mathrm{M}$ LUV suspension with increasing concentrations of pepR. b Normalized $\zeta$-potential dependence on the concentration of pepR. The lines represent the best fit of Eq. 8 to the data. Empty circles and dashed line POPC:POPG 4:1; triangles and solid line POPC:POPG 3:2. Error bars represent the standard deviation from three independent experiments. c Normalized $\zeta$-potential dependence on the concentration of BP100 with a $250-\mu \mathrm{M}$ POPC:POPG 2:1 LUV solution. The line represents the best fit of Eq. 8 to the data. Raw data of $\mathbf{c}$ from (Ferre et al. 2009)

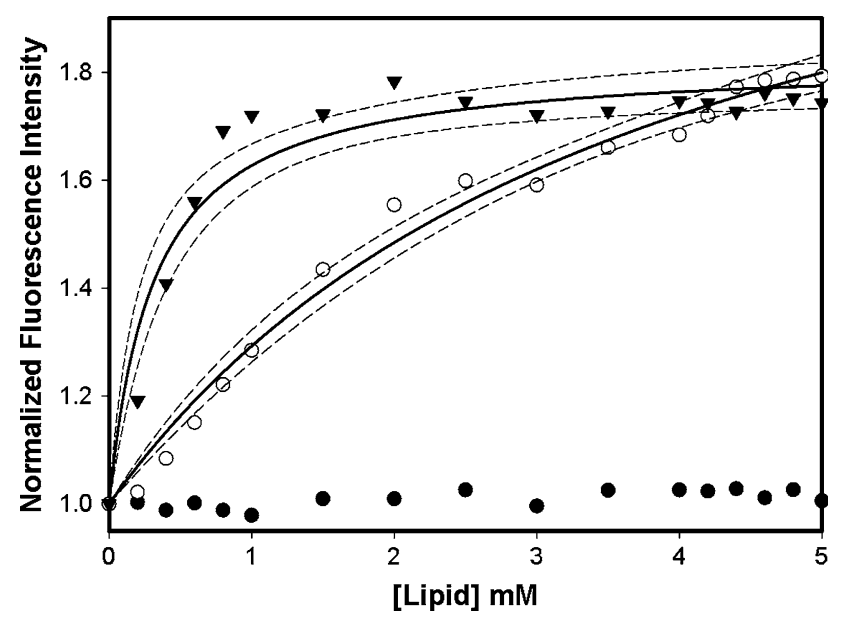

Fig. 3 Determination of the partition constant by fluorescence spectroscopy. Partition curves of a $36-\mu \mathrm{M}$ solution of pepR titrated with different lipid mixtures (LUV). The partition constants were obtained by the non-linear fitting of Eq. 4 to the data. The solid lines represent the non-linear fitting and the dashed lines the 95\% confidence intervals. Filled circles POPC LUVs, empty circles POPC:POPG 4:1 and filled triangles POPC:POPG 3:2

studying the partition of pepR to $20 \%$ charged vesicles the value of $K_{P}$ from $\zeta$-potential does not totally match. This may be related to pepR being quite long and cationic, and the fraction of anionic phospholipids fairly low, which may result in other factors superimposing to simple electrostatic interactions (White et al. 2001; White and Wimley 1998).

$\zeta$-Potential is a powerful approach when studying charged molecules that are also good scatters. The major drawback of this methodology is the necessity of charged particles. Equation 8 shows that if both lipid and peptide have no charge, the equation is not valid anymore to calculate the $K_{p}$. Another assumption that we have to consider when applying this model is that each charge of peptide is responsible for the neutralization of one charge of the lipid. Therefore, a stoichiometry of 1:1 peptide charge:lipid charge is assumed in the model.

When applying the fluorescence spectroscopy-based model, the major requirement is to have a fluorescent reporter in the membrane-active molecule. Many peptides have fluorescent amino acid residues in their sequence, 
Table 1 Values of the partition constants of BP100 and pepR to liposomes with different lipid compositions, at $\mathrm{pH} 7.4$, obtained by $\zeta$-potential and fluorescence spectroscopy measurements. $\left(K_{p} \pm\right.$ standard deviation)

\begin{tabular}{llll}
\hline Peptide & Lipid mixture & \multicolumn{1}{l}{$K_{p} / 10^{3}$} & \\
\cline { 3 - 4 } & & $\begin{array}{l}\text { Fluorescence } \\
\text { spectroscopy }\end{array}$ & $\zeta$-Potential \\
\hline BP100 & POPC:POPG $1: 2$ & $50.7 \pm 9.30$ & $56.7 \pm 0.70$ \\
pepR & POPC:POPG 4:1 & $0.44 \pm 0.08$ & $1.70 \pm 0.06$ \\
& POPC:POPG 3:2 & $4.50 \pm 1.06$ & $5.50 \pm 1.30$ \\
\hline
\end{tabular}

a $\zeta$-Potential data (Ferre et al. 2009)

namely tryptophan, tyrosine or phenylalanine (very low signal), and work as reporters of the surrounding environment (Santos and Castanho 2002a). However, there are some cases where no reporter is available in the native molecule. To circumvent this problem, molecule labeling with a fluorescent probe is very common. However, peptide labeling may change the biophysical and biological properties of the native molecule and modify their interaction with lipid membranes. With this novel approach of determining $K_{p}$ by $\zeta$-potential, it is possible to quantify the partition of molecules with no intrinsic fluorescent reporter, as long as they are charged, which is an almost universal condition in some areas of study, such as antimicrobial peptides.

Acknowledgments The authors thank Rafael Ferre, Marta Planas, Lidia Feliu and Eduard Bardají (Department of Chemistry, University of Girona, Spain) for the synthesis of BP100, and Wioleta Kowalczyk and David Andreu (Department of Experimental and Health Sciences, Pompeu Fabra University, Barcelona, Spain) for the synthesis of pepR. This work was partially supported by project PTDC/QUI/ 69937/2006 from Fundação para a Ciência e Tecnologia-Ministério da Ciência, Tecnologia e Ensino Superior (FCT-MCTES, Portugal), and by Fundação Calouste Gulbenkian (Portugal). JMF and MMD also thank FCT-MCTES for grants IMM/BT/37-2010 and SFRH/BD/ 41750/2007, respectively.

Open Access This article is distributed under the terms of the Creative Commons Attribution Noncommercial License which permits any noncommercial use, distribution, and reproduction in any medium, provided the original author(s) and source are credited.

\section{References}

Alves C, Melo M, Franquelim H, Ferre R, Planas M, Feliu L, Bardají E, Kowalczyk W, Andreu D, Santos N, Fernandes M, Castanho M (2010) Escherichia coli cell surface perturbation and disruption induced by antimicrobial peptides BP100 and pepR. J Biol Chem 285(36):27536-27544. doi:10.1074/jbc.M110.130955

Castanho M, Fernandes M (2006a) Lipid membrane-induced optimization for ligand-receptor docking: recent tools. Eur Biophys J

Castanho MARB, Fernandes MX (2006b) Lipid membrane-induced optimization for ligand-receptor docking: recent tools and insights for the "membrane catalysis" model. Eur Biophys J 35(2):92-103. doi:10.1007/s00249-005-0007-9

Castanho M, Prieto M, Acuña AU (1996) The transverse location of the fluorescent probe trans-parinaric acid in lipid bilayers. Biochim Biophys Acta 1279(2):164-168

Dawson RM, Liu CQ (2008) Properties and applications of antimicrobial peptides in biodefense against biological warfare threat agents. Crit Rev Microbiol 34(2):89-107. doi:10.1080/10408410 802143808

Delgado AV, González-Caballero F, Hunter RJ, Koopal LK, Lyklema J, International Union of Pure and Applied Chemistry PaBCDITR (2007) Measurement and interpretation of electrokinetic phenomena. J Colloid Interface Sci 309(2):194-224. doi:10.1016/ j.jcis.2006.12.075

Domingues MM, Santiago PS, Castanho MARB, Santos NC (2008) What can light scattering spectroscopy do for membrane-active peptide studies? J Pept Sci 14(4):394

Ferre R, Melo MN, Correia AD, Feliu L, Bardaji E, Planas M, Castanho M (2009) Synergistic effects of the membrane actions of cecropin-melittin antimicrobial hybrid peptide BP100. Biophys J 96(5):1815-1827. doi:10.1016/j.bpj.2008.11.053

Hancock RE, Chapple DS (1999) Peptide antibiotics. Antimicrob Agents Chemother 43(6):1317-1323

Kaszuba M, Corbett J, Watson FM, Jones A (2010) High-concentration zeta potential measurements using light-scattering techniques. Philos Trans A Math Phys Eng Sci 368(1927):44394451. doi:10.1098/rsta.2010.0175

Kirby B, Hasselbrink E (2004) Zeta potential of microfluidic substrates: 1. Theory, experimental techniques, and effects on separations. Electrophoresis 25(2):187-202

Ladokhin AS, Jayasinghe S, White SH (2000) How to measure and analyse tryptophan fluorescence in membranes properly, and why bother? Anal Biochem 285(2):235-245

Lopes S, Castanho M (2005) Overview of common spectroscopic methods to determine the orientation/alignment of membrane probes and drugs in lipidic bilayers. Curr Organ Chem 9(9):889-898

Malvern (2007) Zetasizer nano user manual. Malvern Instruments Ltd, Malvern

Matos PM, Franquelim HG, Castanho MARB, Santos NC (2010) Quantitative assessment of peptide-lipid interactions. Ubiquitous fluorescence methodologies. Biochim Biophys Acta 1798(11): 1999-2012. doi:10.1016/j.bbamem.2010.07.012

Mayer LD, Hope MJ, Cullis PR (1986) Vesicles of variable sizes produced by a rapid extrusion procedure. Biochim Biophys Acta 858(1):161-168

Miller JF, Schatzel K, Vincent B (1991) The determination of very small electrophoretic mobilities in polar and nonpolar colloidal dispersions using phase-analysis light-scattering. J Colloid Interface Sci 143(2):532-554

Reddy K, Yedery R, Aranha C (2004) Antimicrobial peptides: premises and promises. Int J Antimicrob Agents 24(6):536-547

Ribeiro MMB, Melo MN, Serrano ID, Santos NC, Castanho MARB (2010) Drug-lipid interaction evaluation: why a 19th century solution? Trends Pharmacol Sci. doi:10.1016/j.tips.2010.06.007

Santos N, Castanho M (2002a) Fluorescence spectroscopy methodologies on the study of proteins and peptides. On the 150th anniversary of protein fluorescence. Trends Appl Spectrosc 4:113-125

Santos N, Castanho M (2002b) Lipossomas: a Bala Magica Acertou? Quim Nova 25:1181-1185

Santos NC, Prieto M, Castanho MARB (2003) Quantifying molecular partition into model systems of biomembranes: an emphasis on optical spectroscopic methods. Biochim Biophys Acta 1612(2): $123-135$

Troeira Henriques S, Nuno Melo M, Castanho M (2007) How to address CPP and AMP translocation? Methods to detect and 
quantify peptide internalization in vitro and in vivo (Review). Mol Membr Biol 24(3):173-184

Tscharnuter W (2001) Mobility measurements by phase analysis. Appl Opt 40:3995-4003
White S, Wimley W (1998) Hydrophobic interactions of peptides with membrane interfaces. Biochim Biophys Acta 1376:339-352

White SH, Ladokhin AS, Jayasinghe S, Hristova K (2001) How membranes shape protein structure. J Biol Chem 276:32395-32398 